OPEN ACCESS

Edited by:

Jiqiang Ling,

University of Maryland, College Park,

United States

Reviewed by:

Ma. Cristina Del Rincón-Castro,

University of Guanajuato, Mexico

Jia Yin,

Hunan Normal University, China

${ }^{*}$ Correspondence:

$\mathrm{Jin} \mathrm{He}$

hejin@mail.hzau.edu.cn

Jieping Wang

wangjpfaas@foxmail.com

Specialty section:

This article was submitted to

Microbial Physiology and Metabolism,

a section of the journal

Frontiers in Microbiology

Received: 09 September 2020 Accepted: 04 November 2020

Published: 26 November 2020

Citation:

Li Z, Zhu L, Yu Z, Liu L, Chou S-H, Wang $J$ and He J (2020) 6S-1 RNA

Contributes to Sporulation and

Parasporal Crystal Formation in

Bacillus thuringiensis.

Front. Microbiol. 11:604458.

doi: 10.3389/fmicb.2020.604458

\section{S-1 RNA Contributes to Sporulation and Parasporal Crystal Formation in Bacillus thuringiensis}

\author{
Zhou Li', Li Zhu', Zhaoqing Yu', Lu Liu', Shan-Ho Chou ${ }^{1}$, Jieping Wang ${ }^{2 *}$ and Jin He ${ }^{1 *}$ \\ 'State Key Laboratory of Agricultural Microbiology, College of Life Science and Technology, Huazhong Agricultural University, \\ Wuhan, China, ${ }^{2}$ Agricultural BioResources Institute, Fujian Academy of Agricultural Sciences, Fuzhou, China
}

6S RNA is a kind of high-abundance non-coding RNA that globally regulates bacterial transcription by interacting with RNA polymerase holoenzyme. Through bioinformatics analysis, we found that there are two tandem 6S RNA-encoding genes in the genomes of Bacillus cereus group bacteria. Using Bacillus thuringiensis BMB171 as the starting strain, we have explored the physiological functions of 6S RNAs, and found that the genes ssrSA and ssrSB encoding 6S-1 and 6S-2 RNAs were located in the same operon and are co-transcribed as a precursor that might be processed by specific ribonucleases to form mature 6S-1 and 6S-2 RNAs. We also constructed two single-gene deletion mutant strains $\Delta s s r S A$ and $\Delta s s r S B$ and a double-gene deletion mutant strain $\triangle s s r S A B$ by means of the markerless gene knockout method. Our data show that deletion of 6S-1 RNA inhibited the growth of $B$. thuringiensis in the stationary phase, leading to lysis of some bacterial cells. Furthermore, deletion of $6 \mathrm{~S}-1$ RNA also significantly reduced the spore number and parasporal crystal content. Our work reveals that $B$. thuringiensis 6S RNA played an important regulatory role in ensuring the sporulation and parasporal crystal formation.

\section{Keywords: non-coding RNA, 6S RNA, Bacillus thuringiensis, sporulation, parasporal crystal formation}

\section{INTRODUCTION}

In 1967, Hindley (1967) first discovered a highly abundant non-coding RNA in Escherichia coli. Because it exhibits a sedimentation coefficient of $6 \mathrm{~S}$, it was thus named 6S RNA, with its nucleotide sequence further determined by Brownlee (1971) afterwards. However, the study of 6S RNA remains silent for a long time until 2000, when Wassarman and Storz (2000) found that E. coli 6S RNA exists in high abundance throughout all bacterial growth phases, and exhibits a concentration as high as 10,000 molecules/cell in E. coli cells in the stationary phase. High abundant $6 \mathrm{~S}$ RNA is found to regulate gene transcription in the stationary phase by combining with $\sigma^{70}$-denpendent RNA polymerase holoenzyme, thus leading to the response regulation of bacterial to stresses such as starvation. Infact, all previous studies also seem to indicate that 6S RNA is a global regulatory factor (Wassarman and Storz, 2000; Cavanagh and Wassarman, 2014; Steuten et al., 2014; Wassarman, 2018). Later on, 6S RNA is found to exist in even more bacteria. Wehner et al. (2014) identified 1,750 6S RNA-encoding genes in 1,611 bacterial genomes, and found that the sizes of 6S RNA-encoding 
genes ranging from 153 to 237 base pairs (with an average of $183 \mathrm{bp}$ ). While most bacteria seem to contain only one 6S RNA-encoding gene in their genomes, some bacteria, such as Bacillus genus in Firmicutes phylum, do contain two $6 \mathrm{~S}$ RNA-encoding genes. Interestingly, even four such $6 \mathrm{~S}$ RNA-encoding genes are found in the genome of Magnetococcus sp. MC- 1 of $\alpha$-Proteobacteria. In fact, 6S RNA-encoding genes are now found to be widely distributed in various bacteria, even in bacteria from the phyla Chloroflexi and Aquificae at the root of the phylogenetic tree (Wehner et al., 2014), indicating that 6S RNAs are originated quite early and are widely distributed.

Besides, Trotochaud and Wassarman (2004) also reveal that the survival ability of $E$. coli is distinctively reduced in the stationary phase when the 6S RNA-encoding gene ssrS1 is deleted, indicating that 6S RNA is beneficial to the normal growth and survival of E. coli in the stationary phase. Indeed, more and more regulatory functions of $6 \mathrm{~S}$ RNA are gradually unveiled after many more in-depth researches. For example, it can now delay the sporulation of Bacillus subtilis (Cavanagh and Wassarman, 2013), promote the photosynthesis of Synechocystis (Heilmann et al., 2017), enhance the gene expression of pathogenicity island in Salmonella enterica serovar Typhimurium (Ren et al., 2017), increase the synthesis of antibiotics in Streptomyces coelicolor (Mikulík et al., 2014), optimize the symbiosis of Bradyrhizobium with leguminous plants (Madhugiri et al., 2012), and so on.

B. subtilis contains two kinds of $6 \mathrm{~S}$ RNAs, called $6 \mathrm{~S}-1$ and 6S-2 RNAs, with their encoding genes $b s r A$ and $b s r B$ located in different regions of the genome. While deletion of $6 \mathrm{~S}-1$ RNA inhibits the growth of $B$. subtilis in the stationary phase (Hoch et al., 2015) and promotes earlier sporulation initiation by accelerating the utilization of nutrients (Cavanagh and Wassarman, 2013), the lack of 6S-2 RNA does not, however, seem to affect the growth and sporulation in the stationary phase (Cavanagh and Wassarman, 2013; Hoch et al., 2015). Until now, its physiological function remains unclear.

The Bacillus cereus group is a taxonomic group comprising closely related species of the Bacillus genus that includes more than 20 different species (Miller et al., 2016; Liu et al., 2017). Among them, the insect pathogen Bacillus thuringiensis, the anthracnose pathogen Bacillus anthracis, and the food-borne opportunistic pathogen B. cereus (Wang et al., 2019), have attracted extensive attention. Different from B. subtilis that harbors two $6 \mathrm{~S}$ RNA-encoding genes located in different regions, $B$. cereus group bacteria contain the 6S RNA-encoding genes arranged in tandem in their genomes (Wehner et al., 2014). In this study, taking the B. thuringiensis BMB171 as a model to study the physiological function of $6 \mathrm{~S}$ RNAs in $B$. cereus group bacteria, we found that the encoding genes $s s r S A$ and $s s r S B$ of $6 \mathrm{~S}-1$ and $6 \mathrm{~S}-2$ RNAs in BMB171 were located in the same operon and were co-transcribed as a precursor, which might be processed by ribonucleases to form mature 6S-1 and 6S-2 RNAs. Furthermore, deletion of the $s s r S A$ gene inhibited the growth of $B$. thuringiensis in the stationary phase and decreased the sporulation and parasporal crystal formation. Besides, we found that diminished sporulation is primarily due to the decreased growth rate of the $s s r S A$ deletion mutant in the stationary phase.

\section{MATERIALS AND METHODS}

\section{Bacterial Strains and Growth Conditions}

The plasmids and strains used in this study are listed in Tables 1, 2, with primers used listed in Supplementary Table S1, respectively. Escherichia coli $\mathrm{DH} 5 \alpha$ was used for cloning experiment and was cultured at $37^{\circ} \mathrm{C}$ in lysogeny broth (LB) medium (g/L: tryptone, 10; yeast extract, 5; and $\mathrm{NaCl}, 10$ ). The medium was adjusted to $\mathrm{pH} 7.0$ before autoclaving at $121^{\circ} \mathrm{C}$ for $15 \mathrm{~min}$. Bacillus thuringiensis BMB171 and its derivative strains were cultured at $28^{\circ} \mathrm{C}$ in the GYS medium (g/L: glucose, 1.00; yeast extract, 2.00; $\mathrm{K}_{2} \mathrm{HPO}_{4} \cdot 3 \mathrm{H}_{2} \mathrm{O}, 0.66$; $\left(\mathrm{NH}_{4}\right)_{2} \mathrm{SO}_{4}, 2.00 ; \mathrm{MgSO}_{4} \cdot 7 \mathrm{H}_{2} \mathrm{O}, 0.04 ; \mathrm{MnSO}_{4} \cdot \mathrm{H}_{2} \mathrm{O}, 0.04$; and $\mathrm{CaCl}_{2}, 0.08$ ), with the medium autoclaved at $115^{\circ} \mathrm{C}$ for $30 \mathrm{~min}$ after $\mathrm{pH}$ value being adjusted to 7.8. When necessary, relevant antibiotics were added to the cultures with the following final concentration: $50 \mu \mathrm{g} / \mathrm{ml}$ kanamycin, $25 \mu \mathrm{g} / \mathrm{ml}$ erythromycin, $100 \mu \mathrm{g} / \mathrm{ml}$ ampicillin, $300 \mu \mathrm{g} / \mathrm{ml}$ spectinomycin, or $60 \mathrm{U}$ polymyxin (Wang et al., 2019). For the decoyinineadded experiment, cells were grown to an $\mathrm{OD}_{600}$ of 0.5 in GYS medium, followed by the addition of decoyinine to $0.4 \mathrm{mg} / \mathrm{ml}$ as previously described (Mitani et al., 1977; Ikehara et al., 1982; Cavanagh and Wassarman, 2013).

\section{RNA Extraction and RT-qPCR}

The samples of $30 \mathrm{ml}$ each from BMB171 and its derivative strains were cultured in GYS medium for $11 \mathrm{~h}$ before being centrifuged, followed by total RNA extraction and RT-qPCR experiments as previously described (Zheng et al., 2015, 2020; Fu et al., 2018). In these experiments, the gapdh gene was used as an internal control.

\section{Identification of Transcription Start Site}

The 5'-rapid amplification of complementary DNA (cDNA) ends (5'-RACE) experiment was performed to identity the trancription start site (TSS) as described previously with some modifications (Ali et al., 2017). RNA was first extracted from BMB171 cells that were grown in GYS, followed by reverse transcription to cDNA. The 3 '-end of cDNA was then labeled by poly $(\mathrm{dA})$ using terminal deoxynucleotidyl transferase (Takara, Japan). The cDNA was then PCR amplified using primers of Primer-8 and $s s r S B-R$ as listed in Supplementary Table S1. The PCR products were then cloned to the pMD19-T vector (Takara, Japan) and sequenced (Wang et al., 2019).

\section{Determination of $\beta$-Galactosidase Activity}

BMB171/pHT1K, BMB171/P1-lacZ, and BMB171/P2-lacZ strains were grown at $28^{\circ} \mathrm{C}$ in a shaking incubator at $200 \mathrm{rpm}$ in $100 \mathrm{ml} \mathrm{GYS}$ with $25 \mu \mathrm{g} / \mathrm{ml}$ erythromycin. Two milliliters of each culture was separately collected at indicated time and assayed for $\beta$-galactosidase activity as described previously (Zhou et al., 2017; Wang et al., 2019).

\section{Spore Count by Spread-Plate Method}

BMB171 and its single deletion 6S-1 RNA mutant $\Delta s s r S A$, single deletion 6S-2 RNA mutant $\Delta s s r S B$, and the double deletion mutant 
TABLE 1 | Plasmids used in this study.

\begin{tabular}{|c|c|c|c|}
\hline Plasmids & Relevant characteristics & Purposes & Origins \\
\hline pHT1K & B. thuringiensis-E. coli shuttle plasmid; $\mathrm{Amp}^{R} \mathrm{Erm}^{\mathrm{R}}$ & $\begin{array}{l}\text { For } \beta \text {-galactosidase assays and gene } \\
\text { complementation }\end{array}$ & Wang et al., 2013a \\
\hline pHT1K-lacZ & $\begin{array}{l}\text { pHT1K plasmid carrying the promoter-less lac } Z \text { gene, for } \beta \text {-galactosidase } \\
\text { activity assay }\end{array}$ & $\beta$-galactosidase assays & Wang et al., 2013a \\
\hline pHT1K-P1-lacZ & $\begin{array}{l}\text { pHT1K carrying the promoter region }(-190 \text { to }+10) \text { of ssrS operon fused } \\
\text { with lacZ }\end{array}$ & $\beta$-galactosidase assays & This work \\
\hline pHT1K-P2-lacZ & $\begin{array}{l}\text { pHT1K carrying the upstream region }(+10 \text { to }+214) \text { of } s s r S B \text { fused with } \\
\text { lacZ }\end{array}$ & $\beta$-galactosidase assays & This work \\
\hline pHT1K-P1-ssrSA & $\begin{array}{l}\text { pHT1K carrying the promoter and ssrSA encoding region of } s s r S \text { operon } \\
\text { fused with terminator region of } s s r S \text { operon }\end{array}$ & Gene complementation & This work \\
\hline pHT1K-P1-sSrSAB & pHT1K carrying the promoter and encoding region of the ssrS operon & Gene complementation & This work \\
\hline pRP1028 & $\begin{array}{l}\text { B. thuringiensis-E. coli shuttle plasmid; } \mathrm{Amp}^{\mathrm{R}} \mathrm{Erm}^{\mathrm{R}} \text {; carrying turbo-rfp gene } \\
\text { and an I-Scel recognition site }\end{array}$ & Gene deletion & Janes and Stibitz, 2006 \\
\hline pSS4332 & $\begin{array}{l}\text { B. thuringiensis- } E \text {. coli shuttle plasmid; } \mathrm{Km}^{\mathrm{R}} \text {; carrying gfp and I-Scel } \\
\text { restriction enzyme encoding gene }\end{array}$ & Gene deletion & Janes and Stibitz, 2006 \\
\hline pSS1827 & The helper plasmid for conjugative transfer; $\mathrm{Amp}^{R}$ & Gene deletion & Janes and Stibitz, 2006 \\
\hline pRP1028-ssrSA-UD & $\begin{array}{l}\text { pRP1028 with the upstream and downstream regions of ssrSA, an } \\
\text { intermediate plasmid in gene deletion experiment }\end{array}$ & Gene deletion & This work \\
\hline pRP1028-ssrSB-UD & $\begin{array}{l}\text { pRP1028 with the upstream and downstream regions of } s s r S B \text {, an } \\
\text { intermediate plasmid in gene deletion experiments }\end{array}$ & Gene deletion & This work \\
\hline pRP1028-ssrSAB-UD & $\begin{array}{l}\text { pRP1028 with the upstream region of } S S r S A \text { and downstream region of } \\
\text { ssrSB, an intermediate plasmid in gene deletion experiments }\end{array}$ & Gene deletion & This work \\
\hline pBMB43-304 & $\begin{array}{l}\text { B. thuringiensis-E. coli shuttle plasmid; Amp }{ }^{R} \mathrm{Erm}^{\mathrm{R}} \text {; carrying ORF of } \\
\text { cry1Ac10; }\end{array}$ & $\begin{array}{l}\text { Determination of the parasporal } \\
\text { crystal protein Cry1Ac } 10\end{array}$ & Qi et al., 2015 \\
\hline
\end{tabular}

TABLE 2 | Strains used in this study.

\begin{tabular}{|c|c|c|}
\hline Strains & Relevant characteristics & Origins \\
\hline E. coli DH5 $\alpha$ & F-Ф80lacZuM15 $\Delta$ (lacZYA-argF) U169 recA1 endA1 hsdR17 (rk-, mk+) phoA supE44 thi-1 gyrA96 relA1 $\lambda$ - & Beijing TransGen Biotech Co., Ltd. \\
\hline BMB171 & B. thuringiensis strain BMB171; an acrystalliferous mutant strain; high transformation frequency & He et al., 2010; Wang et al., 2013a \\
\hline$\Delta s s r S A$ & Markerless deletion of ssrSA in BMB171 & This work \\
\hline$\Delta s s r S B$ & Markerless deletion of ssrSB in BMB171 & This work \\
\hline$\Delta s s r S A B$ & Markerless deletion of ssrSA and ssrSB in BMB171 & This work \\
\hline BMB171/pHT1K & BMB171 strain harboring plasmid pHT1K & This work \\
\hline$\Delta s s r S A / P 1-s s r S A$ & Gene complemented strain: $\Delta$ ssrSA strain harboring plasmids pHT1K-P1-ssrSA & This work \\
\hline$\Delta s s r S A B / P 1-s s r S A B$ & Gene complemented strain: $\triangle s s r S A B$ strain harboring plasmid pHT1K-P1-ssrSAB & This work \\
\hline BMB171/lacZ & BMB171 strain harboring pHT1K carrying the promoter-less lac Z gene & This work \\
\hline BMB171/P1-lacZ & BMB171 strain harboring pHT1K carrying the promoter region of ssrSA fused with lac $Z$ & This work \\
\hline BMB171/P2-lacZ & BMB171 strain harboring pHT1K carrying the upstream region of ssrSB fused with lac $Z$ & This work \\
\hline BMB171-cry & BMB171 strain harboring plasmid pBMB43-304 & This work \\
\hline$\Delta s s r S A-c r y$ & $\Delta s s r S A$ strain harboring plasmid pBMB43-304 & This work \\
\hline$\Delta$ ssrSB-cry & $\Delta s s r S B$ strain harboring plasmid pBMB43-304 & This work \\
\hline$\Delta s s r S A B$-cry & $\Delta s s r S A B$ strain harboring plasmid pBMB43-304 & This work \\
\hline
\end{tabular}

$\triangle s s r S A B$ were cultured in GYS medium at $28^{\circ} \mathrm{C}$ for $24 \mathrm{~h}$, which were then heated to $65^{\circ} \mathrm{C}$ for $30 \mathrm{~min}$, followed by gradient dilution (10 times) with M9 minimum medium. Around $100 \mu \mathrm{l}$ of each diluent was then spread onto LB plates. The colony-forming units (CFUs) per ml were then counted (Wang et al., 2016).

\section{Phase-Contrast Microscopic Analysis of Sporulation}

BMB171 and its derivative strains were cultured at $28^{\circ} \mathrm{C}$ in GYS medium. To observe the morphology of vegetative cells and spores, $5 \mu \mathrm{l}$ of each cell sample was collected at indicated time points, spotted onto the center of a glass slide, and covered with a coverslip. Spores were then observed with a phasecontrast microscope (Olympus, Japan; Zheng et al., 2020).

\section{Transmission Electron Microscope}

To observe the morphology of BMB171 cells and its derivative strains, $4 \mathrm{ml}$ of each sample was harvested by centrifugation at $17 \mathrm{~h}$, with the cell pellets resuspended in $2.5 \%$ glutaraldehyde, and stored at $4{ }^{\circ} \mathrm{C}$ overnight. Ultra-thin sections were finally prepared and stained as described (Craig et al., 1997). A Hitachi H-7000 FA transmission electron microscope (Hitachi, Japan) was then used for observation.

\section{Construction of Markerless Gene Deletion Strains}

The markerless gene deletion mediated by homing endonuclease I-SceI was performed in B. thuringiensis as previously reported (Zheng et al., 2015; Tang et al., 2016; Wang et al., 2016). 
Intermediate plasmids (pRP1028-ssrSA-UD, pRP1028-ssrSB-UD, and pRP1028-ssrSAB-UD) used in this study for gene deletion experiments were listed in Table 1. The helper plasmid pSS1827 for conjugational transfer and B. thuringiensis-E. coli shuttle plasmid pRP1028 carrying an I-SceI recognition site and pSS4332 carrying I-Scel restriction enzyme encoding gene was listed in Table $\mathbf{1}$.

\section{Observation of Parasporal Crystal and Determination of Parasporal Crystal Protein}

Crystalliferous strains of BMB171-cry, $\Delta s s r S A-c r y, \Delta s s r S B-c r y$, and $\triangle$ ssrSAB-cry were obtained by transformation of the cry $1 A c 10$ gene with its original promoter in the plasmid pBMB43-304 (Qi et al., 2015) into BMB171, $\Delta s s r S A, \Delta s s r S B$, and $\Delta s s r S A B$ strains, respectively. To observe parasporal crystals, the crystalliferous strains were grown at $28^{\circ} \mathrm{C}$ and $200 \mathrm{rpm}$ for $24 \mathrm{~h}$ in GYS medium supplemented with $25 \mathrm{mg} / \mathrm{ml}$ erythromycin. One drop from each culture was spotted onto the center of a glass slide, and covered with a coverslip. Parasporal crystals were then observed with a phase-contrast microscope (Olympus, Japan). To extract Cry1Ac10 protein, each culture was collected separately by centrifugation at 6,000 $g$ for $15 \mathrm{~min}$ (AG Eppendorf, Hamburg, Germany). Procedure for the separation of Cry1Ac10 protein was carried out according to a previous study (Wang et al., 2013b). Finally, the Cry1Ac10 protein was visualized by SDS-PAGE, with its concentration measured by the Bradford method (Wang et al., 2016).

\section{RESULTS}

\section{The ssrSA and ssrSB Genes Were Located in the Same Operon and Were Co-Transcribed}

The BMB171 strain was found to possess two 6S RNAs, with their encoding genes ssrSA (BMB171_RS29145) and ssrSB (BMB171_RS291506) located in tandem in the genome (Figure 1A). To explore whether the ssrSA and $s s r S B$ genes are within the same operon, we first verified the co-transcription of the ssrSA and $s s r S B$ genes by the semiquantitative reverse transcription PCR (SqRT-PCR; Supplementary Figure $\mathbf{S 1}$ ), and found they were indeed co-transcribed. To further confirm that the $s s r S B$ gene is not transcribed individually, we carried out the $\beta$-galactosidase assays to detect the promoter activities of the upstream regions of $s s r S A$ and $s s r S B$ genes in the BMB171 genome, respectively. We found that the upstream sequence of the ssrSA gene (P1 region) exhibited strong transcription initiation activities in the logarithmic phase $(5 \mathrm{~h})$, transition phase $(11 \mathrm{~h})$, and stationary phase $(17 \mathrm{~h})$, while those of the $s s \mathrm{sB}$ gene (P2 region) basically exhibited no such transcription initiation activities at all when compared to the no promoter control (Figure 1B). These data indicate that the $s s r S B$ gene was co-transcribed along, but not individually, with ssrSA. Finally, through the 5'-RACE experiment (Figure 2A), we identified a TSS as well as the canonical -35 and -10 regions (Figure 2B) upstream of the $s s r S A$ gene, but found no independent TSS upstream of the $s s r S B$ gene. These results
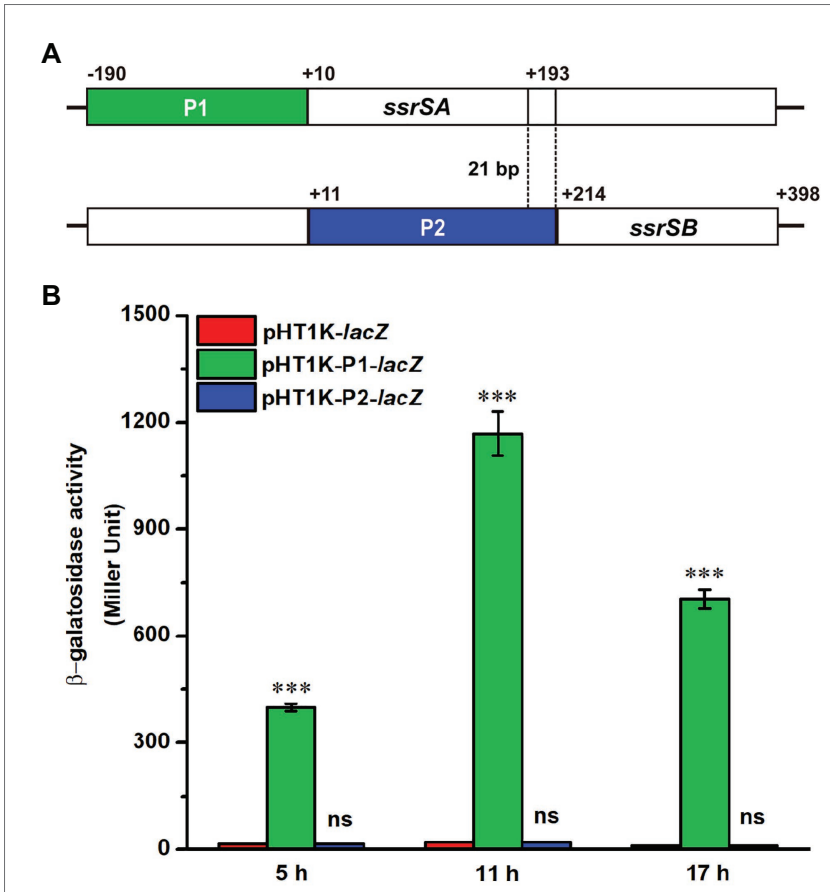

FIGURE 1 | Promoter activity assays of the ssrSA and ssrSB genes in BMB171. (A) Schematic diagram of SsrSA and ssrSB genes and their putative promoter regions of P1 (marked in green) and P2 (marked in blue). The P1 and $\mathrm{P} 2$ regions were each fused with a $\beta$-galactosidase gene (lacZ) and transformed into the plasmid $\mathrm{pHT} 1 \mathrm{~K}$, respectively. (B) The $\beta$-galactosidase activity assay of strains BMB171/P1-lacZ and BMB171/P2-lacZ harboring plasmids pHT1K-P1-lacZ and pHT1K-P2-lacZ with the BMB171/-lacZ harboring $\mathrm{pHT} 1 \mathrm{~K}$ carrying a promoter-less lac $Z$ as the control. The strains were cultured at $28^{\circ} \mathrm{C}$ in GYS medium and were determined in the logarithmic phase $(5 \mathrm{~h})$, transition phase $(11 \mathrm{~h})$, and stationary phase $(17 \mathrm{~h})$. The values were means \pm SDs for triplicate assays. Significances of differences by Student's $t$-test are indicated. ${ }^{* * *} p<0.001 ;{ }^{* *} p<0.01 ;{ }^{*} p<0.05 ;$ ns, $p>0.05$.

thus clearly confirmed that the 6S RNA-encoding genes ssrSA and $s s r B$ were co-transcribed in BMB171.

\section{Deletion of 6S-1 RNA Inhibited the Growth of $B$. thuringiensis in the Stationary Phase}

It has been reported that deletion of 6S-1 RNA results in inhibition of the growth of $B$. subtilis in the stationary phase (Hoch et al., 2015). We thus wonder whether deletion of 6S RNAs also exhibits the similar effect on the growth of $B$. thuringiensis. We have thus used the markerless gene knockout technology to precisely delete the two 6S RNA-encoding genes in the starting strain $\mathrm{BMB} 171$, and constructed the single deletion 6S-1 RNA mutant $\Delta s s r S A$, single deletion 6S-2 RNA mutant $\Delta s s r S B$, and the double deletion mutant $\triangle s s r S A B$. We first determined the growth curves of BMB171 and its mutants, and found that the growth rates of mutants $\Delta s s r S A$ and $\triangle s s r S A B$ declined much more rapidly than that of starting strain BMB171 in the stationary phase, while $\Delta s s r S B$ exhibited no significant change compared to BMB171 (Figure 3A). Next, we checked the cell morphologies of BMB171, $\Delta s s S A$, $\Delta s s r S B$, and $\Delta s s r S A B$ at $17 \mathrm{~h}$ with transmission electron 


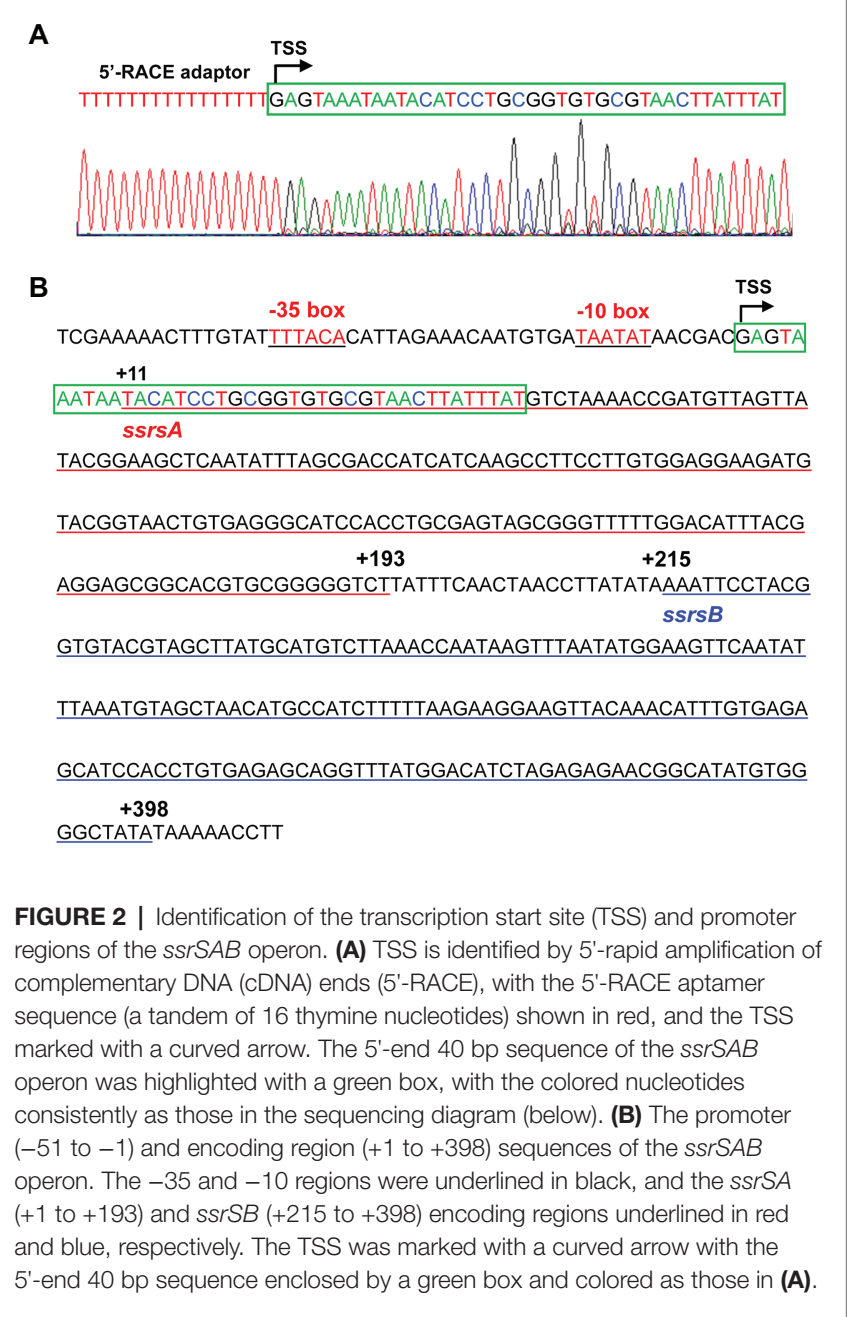

microscope, and found that deletion of 6S-1 RNA caused more cells to lyse (Figure 3B), while the complemented strains $\Delta s s r S A / \mathrm{P} 1-s s r S A$ and $\Delta s s r S A B / \mathrm{P} 1-s s r S A B$ showed no significant differences in bacterial growth and morphology (Supplementary Figure S2) compared to the control strain BMB171/pHT1K. Taken together, these results indicate that deletion of 6 S-1 RNA but not 6S-2 RNA inhibited the growth of $B$. thuringiensis in the stationary phase.

\section{Deletion of 6S-1 RNA Inhibited the Sporulation and Parasporal Crystal Formation}

To investigate the roles of $6 \mathrm{~S}-1$ and $6 \mathrm{~S}-2$ RNAs in the process of sporulation, we first checked the spores of BMB171 and its mutants in the stationary phase through a phase-contrast microscope, and found that the spores formed by mutants $\Delta s s r S A$ and $\triangle s s r S A B$ were much fewer than those of the starting strain BMB171. However, the spore amount of $\Delta s s r S B$ was similar to that of BMB171 (Figure 4A). Second, we counted the spores by spread-plate method, and found that the spore numbers

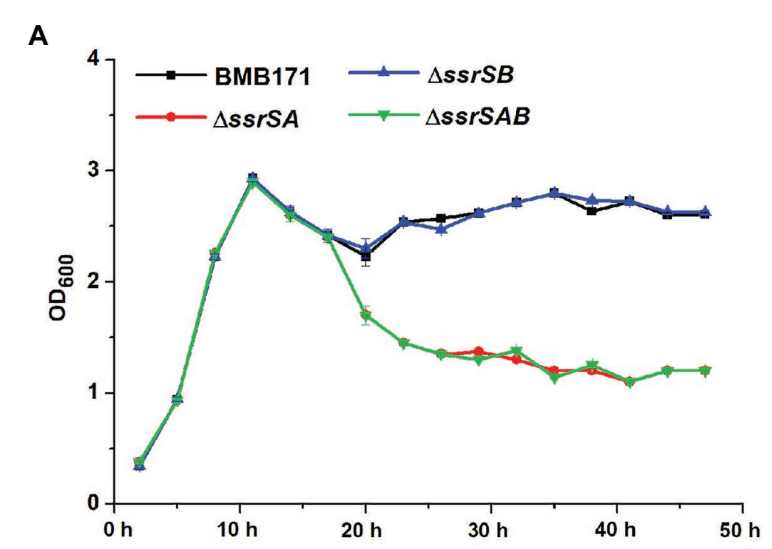

B
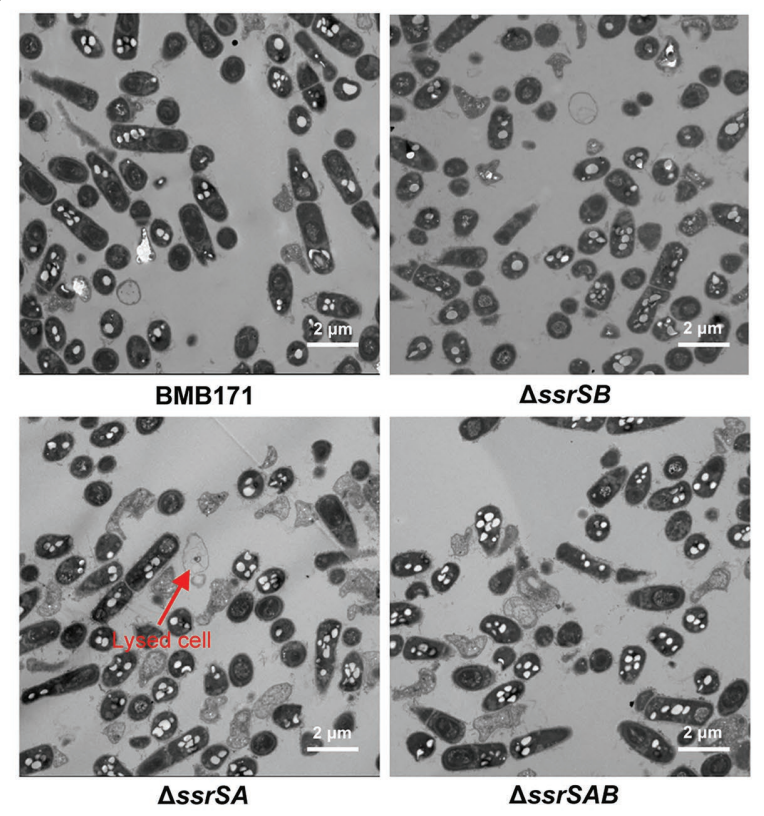

FIGURE 3 | The effect of deleting of 6S RNA-encoding genes on the growths of Bacillus thuringiensis. (A) Determination of the growth curves of BMB171 and its 6S RNA-encoding genes deletion mutants $\Delta s s r S A, \Delta s s r S B$, and $\Delta s s r S A B$. (B) Transmission electron microscope images of the cell morphologies of BMB171 and its deletion mutants $\Delta s s r S A, \Delta s s r S B$, and $\Delta s s r S A B$ at $17 \mathrm{~h}$ (stationary phase). Lysed cells are indicated by red arrows. The above-mentioned strains were cultured at $28^{\circ} \mathrm{C}$ in GYS medium. The values were means \pm SDs for triplicate assays.

formed by $\triangle s s r S A$ and $\triangle s s r S A B$ were much fewer than those of BMB171, whereas the spore number in $\Delta s s r S B$ did not differ much from that in BMB171 (Figure 4B). Meanwhile, we also complemented back the $s s r S A$ and $s s r S A B$ genes into the mutants $\triangle s s r S A$ and $\triangle s s r S A B$, respectively. Phase-contrast microscopy observation and spore count did show that the sporulation capabilities of the complemented strains $\Delta s s r S A / \mathrm{P} 1-s s r S A$ and $\triangle s s r S A B / \mathrm{P} 1-s s r S A B$ have returned to the original level of the control strain BMB171/pHT1K (Supplementary Figure S3). These results indicate that deletion of the $s s r S A$ gene, but not $s s r S B$, inhibited sporulation; namely, the presence of 6S-1 RNA was required for the normal sporulation. 


\section{A}

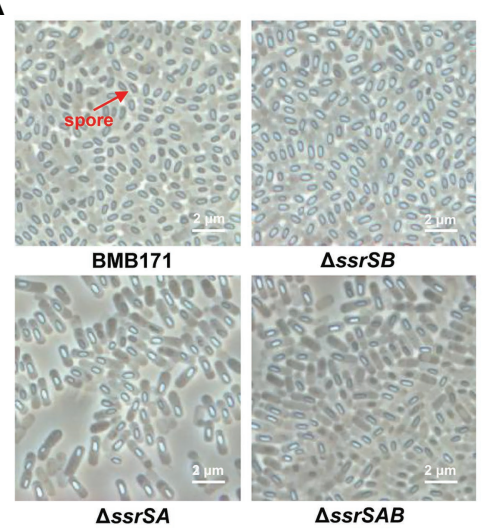

B

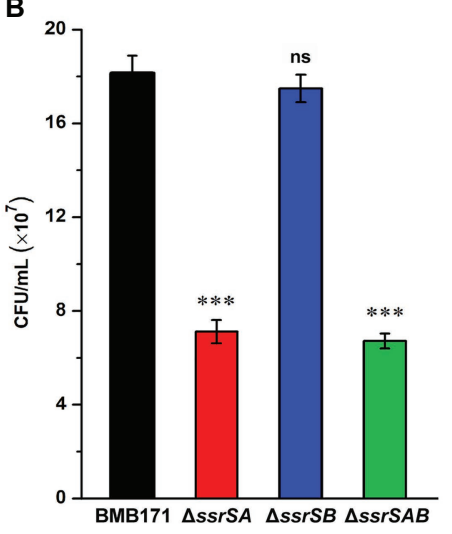

FIGURE 4 | The effect of deleting of 6S RNA-encoding genes on the B. thuringiensis sporulation. (A) Observation of the spore formed by BMB171 and its deletion mutants $\Delta s s r S A, \Delta s s r S B$, and $\Delta s s r S A B$ at $24 \mathrm{~h}$ (stationary phase) using a phase contrast microscope. Oval-shaped spores are indicated by red arrows. (B) The spore counts of BMB171 and its mutants $\Delta s s r S A, \Delta s s r S B$, and $\Delta s s r S A B$ at $24 \mathrm{~h}$ (stationary phase). The strains were cultured at $28^{\circ} \mathrm{C}$ in $\mathrm{GYS}$ medium. The values were means \pm SDs for triplicate assays. Significances of differences by Student's $t$-test are indicated. ${ }^{* * *} p<0.001 ;{ }^{* *} p<0.01 ;{ }^{*} p<0.05 ;$ ns, $p>0.05$.

BMB171 is an acrystalliferous mutant of wild-type YBT-1463 (Li et al., 2000; Qi et al., 2015). To explore whether 6S RNAs affect the parasporal crystal formation, we introduced a pBMB43-304 plasmid containing the parasporal crystal protein encoding gene $c r y 1 A c 10$ and its original promoter sequence (Qi et al., 2015) into the BMB171 and its mutant strains $\Delta s s r S A, \Delta s s r S B$, and $\Delta s s r S A B$, respectively, to obtain the crystalliferous strains of BMB171-cry, $\Delta s s r S A$-cry, $\Delta s s r S B$-cry, and $\Delta s s r S A B$-cry. We first checked the parasporal crystal formation of BMB171 and its mutants in the stationary phase through a phase-contrast microscope, and found that the amounts of parasporal crystals formed by the $\triangle$ ssrSA-cry and $\triangle s s r S A B$-cry strains were far less than that of BMB171cry, while the amount of crystals in the $\Delta s s r S B$-cry was similar to that of BMB171-cry (Figure 5A). Subsequently, we measured the content of parasporal crystal protein CrylAc10, and found that the concentrations of Cry1Ac10 in $\Delta s s r S A$-cry and $\Delta s s r S A B$-cry were remarkably reduced compared to BMB171-cry, while the $\Delta s s r S B$-cry exhibited no substantial difference (Figures 5B,C). These experiments show that deletion of 6S-1 RNA not only inhibited the sporulation of bacterial cells, but also repressed the parasporal crystal formation.

\section{Reduction in Sporulation Capability Was Due to Inhibition of Bacterial Growth in the Stationary Phase}

To figure out the reasons accounting for inhibition of sporulation in BMB171 after 6S-1 RNA deletion, we used the real-time quantitative PCR (RT-qPCR) to examine the transcription levels of several key early sporulation-related genes in BMB171 and its mutant's strains $\triangle s s r S A, \Delta s s r S B$, and $\triangle s s r S A B$. These relevant genes include spo0A, which encodes an essential transcriptional regulatory factor in the initial stage of sporulation (Lopez et al., 2009); kinA, which encodes a phosphorylation kinase that is mainly responsible for the phosphorylation of Spo0A (Jiang et al., 2000a); and spooH, which encodes $\sigma^{\mathrm{H}}$ to promote the transcription of spo0A and kinA (Predich et al., 1992). Taking gene gapdh encoding glyceraldehyde-3-phosphate dehydrogenase as an internal control, we demonstrated that the transcription levels of spoOA, kinA, and spoOH from mutants $\triangle$ ssrSA, $\triangle s s r S B$, and $\triangle s s r S A B$ at $0.5,1.0,1.5$, and $2.0 \mathrm{~h}$ showed almost no difference to those of BMB171 (Supplementary Figure S4). Besides, we further determined the transcriptomes of BMB171 and $\triangle s s r S A B$ in the stationary phase and found that the transcription levels of sporulation-related genes did not change significantly between BMB171 and $\triangle s s r S A B$ (Supplementary Table S2). These data demonstrate that deletion of $6 \mathrm{~S}-1$ RNA did not change the start time of sporulation at the molecular level.

Since deletion of 6S-1 RNA did inhibit the growth of $B$. thuringiensis in the stationary phase, we wonder whether such inhibition also affects their sporulation? Because BMB171 and its various mutants $\Delta s s r S A, \Delta s s r S B$, and $\triangle s s r S A B$ exhibited no obvious difference in the growth curves in the logarithmic phase, we wonder whether there is difference in sporulation efficacy when these strains were induced to produce spores at this time? Given that decoyinine can induce Bacillus cells to produce spores in advance (Cavanagh and Wassarman, 2013; Wang et al., 2016; Zhou et al., 2017), we thus conducted an experiment by adding decoyinine to the GYS medium in the logarithmic phase. The results showed that the spore numbers formed by $\triangle s s r S A, \Delta s s r S B$, and $\triangle s s r S A B$ did not differ much from that of BMB171 (Figure 6). This experiment proves that the reduced sporulation efficacy was mainly caused by inhibition of the growth of $B$. thuringiensis in the stationary phase, not in the logarithmic phase. Further, we found that the transcription levels of genes involved in carbohydrate transport and metabolism, nucleotide transport and metabolism, protein 
A

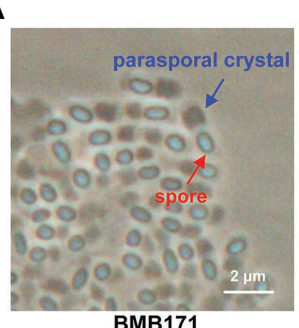

BMB171

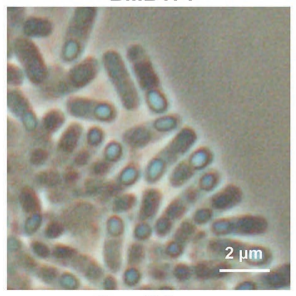

$\Delta$ ssrSA-cry

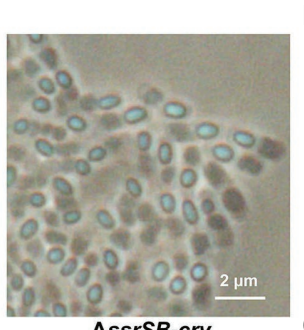

$\Delta$ ssrSB-cry

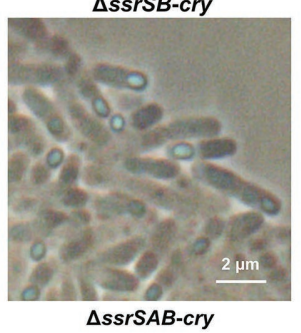

B

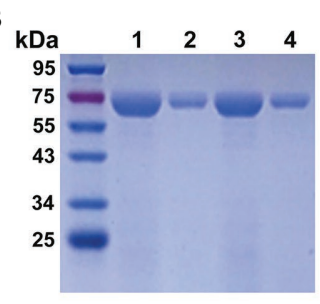

C

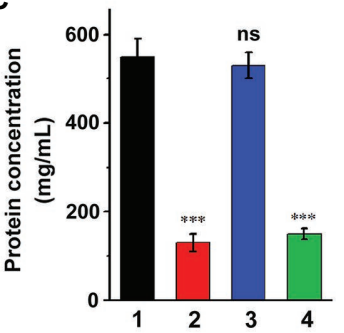

FIGURE 5 | The effect of deleting 6S RNA-encoding genes on the parasporal crystal formation of $B$. thuringiensis (A) Phase contrast microscopy image of the parasporal crystal formation in crystalliferous strains BMB171-cry, $\Delta s s r S A$-cry, $\Delta s s r S B$-cry, and $\Delta s s r S A B$-cry at $24 \mathrm{~h}$ (stationary phase). Cells with parasporal crystals and spores are indicated by blue and red arrows, respectively. (B) Separation of Cry1Ac10 from the four strains by SDS-PAGE. Lines 1-4 represent BMB171-cry, $\Delta$ ssrSA-cry, $\Delta$ ssrSB-cry, and $\Delta s s r S A B$-cry, respectively. (C) The concentrations of Cry1Ac10 in BMB171-cry (1), $\Delta$ ssrSA-cry (2), $\Delta$ ssrSB-cry (3), and $\Delta s s r S A B$-cry (4) at $24 \mathrm{~h}$ (stationary phase). The above-mentioned strains were cultured at $28^{\circ} \mathrm{C}$ in GYS medium. The values were means \pm SDs for triplicate assays. Significances of differences by Student's $t$-test are indicated. ${ }^{* * *} p<0.001 ;{ }^{* *} p<0.01 ;{ }^{*} p<0.05 ;$ ns, $p>0.05$.

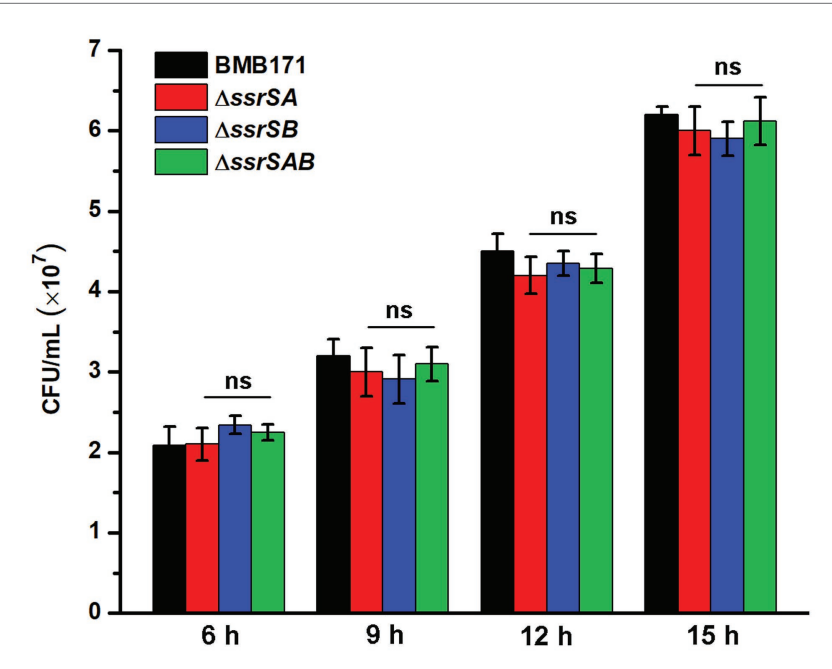

FIGURE 6 | The effect of deleting 6S RNA-encoding genes on the sporulation of BMB171 and its mutants in the logarithmic phase. After adding the sporulation inducer decoyinine $(0.4 \mathrm{mg} / \mathrm{ml})$ to the GYS cultures of abovementioned strains in the logarithmic phase $\left(3 \mathrm{~h}, \mathrm{OD}_{600} \approx 0.5\right)$, the spore numbers were counted at $6,9,12$, and $15 \mathrm{~h}$ after induction. The strains were cultured at $28^{\circ} \mathrm{C}$ and the numbers are revealed as means \pm SDs in triplicate assays. Significances of differences by Student's $t$-test are indicated. ${ }^{\star \star \star} p<0.001 ;{ }^{\star \star} p<0.01 ;{ }^{\star} p<0.05 ;$ ns, $p>0.05$.

translation, and energy production and conversion decreased significantly in $\triangle s s r S A B$ (Supplementary Table S3). Therefore, the decreased growth rate of the ssrSA deletion mutant in the stationary phase is the main reason for the diminished sporulation of $B$. thuringiensis.

\section{DISCUSSION}

In this study, we verified that the $6 \mathrm{~S}-1$ and $6 \mathrm{~S}-2$ RNAs-encoding genes $s s r S A$ and $s s r S B$ were located in the same operon and co-transcribed in $B$. thuringiensis. Through the phenotypic study of BMB171 and its deletion mutants of $\Delta s s r S A, \Delta s s r S B$, and $\Delta s s r S A B$, we found that deletion of $6 \mathrm{~S}-1$ RNA not only inhibited the growth of $B$. thuringiensis in the stationary phase, but also decreased the sporulation and parasporal crystal formation. We further confirmed that inhibition of the growth in the stationary phase is likely the primary reason for the reduced sporulation efficacy.

In BMB171, we validated that the $6 \mathrm{~S}-1$ and $6 \mathrm{~S}-2$ RNAsencoding genes $s s r S A$ and $s s r S B$ were arranged in tandem and co-transcribed, and verified that the upstream sequence of the $s s r S A$ gene exhibited the transcription initiation activity, but not the upstream sequence of the $s s r S B$ gene (Figure 1). These results indicate that the transcriptions of $s s r S A$ and $s s r S B$ genes were initiated through the upstream promoter region of the $s s r S A$ gene, and were jointly transcribed into a long RNA precursor. In $E$. coli, the 6S RNA-encoding gene is first transcribed into a long RNA precursor, which is then cleaved by RNase $\mathrm{E}$ and RNase G into mature 6S RNA (Kim and Lee, 2004). Given the fact that RNase E and RNase G prefer to cut the regions enriched in nucleotides A and U on single-stranded RNA (Mackie, 1998; Jiang et al., 2000b; Tock et al., 2000), so we analyzed the RNA precursor sequence in $\mathrm{BMB} 171$ and found that, similar to E. coli, the 5'- and 3'-ends of the precursor RNA of 6S-1 RNA and 6S-2 RNA in BMB171 are also rich in $A$ and $U$ (Supplementary Figure S5). Moreover, transcriptomic data showed that 6S RNAs existed mainly as two separate mature 6S-1 and 
6S-2 RNAs (Supplementary Figure S6). Combining the above results (Supplementary Figures S1, S5, S6), we suggest that a long precursor 6S RNA is first transcribed, followed by processing with RNase E, RNase G, or other ribonucleases to form mature and functional $6 \mathrm{~S}-1$ and $6 \mathrm{~S}-2$ RNAs.

In the present manuscript, we have analyzed the $6 \mathrm{~S}$ RNA-encoding genes of Bacillus genus bacteria and their gene organization. Bacillus genus bacteria usually contain two different 6S RNA-encoding genes of ssrSA and ssrSB (Supplementary Tables S4-S6). However, unlike other Bacillus genus bacteria that have these two genes located in different regions of the genome, the two 6S RNA-encoding genes of $B$. cereus group bacteria were located in tandem positions. Furthermore, we also verified that they were in the same operon and are transcribed together. The ssrSA and $s s r S B$ genes of $B$. cereus group bacteria are located between the genes encoding thiocyanase and glutathione spermidine synthase. The former is related to the detoxification of exogenous toxins while the latter involved in the resistance to stress such as oxidation (Supplementary Table S4). Other than the B. cereus group, there is also a $B$. subtilis group bacteria composed of more than 20 important bacteria species, such as, B. subtilis, Bacillus licheniformis, Bacillus pumilus, Bacillus velezensis, and Bacillus amyloliquefaciens in Bacillus genus (Caulier et al., 2019; Fayad et al., 2019). Unlike the B. cereus group bacteria, the ssrSA gene of $B$. subtilis group bacteria was mainly located between the FMN-dependent NADH-azoreductase and DNA helicase RecQ encoding genes. The former is related to the detoxification of exogenous toxins, while the latter is linked with DNA repair, recombination, and replication. The $s s r S B$ gene, on the other hand, was mainly located between the aspartate-tRNA ligase and tRNA threonylcarbamoyladenosine dehydratase, both of which were associated with protein translation (Supplementary Table S5). Among Bacillus genus bacteria excluding $B$. cereus group and B. subtilis group, only the aspartatetRNA ligase encoding gene locus upstream of the $s s r S B$ gene was relatively conservative (Supplementary Table S6).

The 6S RNAs in B. cereus group bacteria were quite different to those of $B$. subtilis group bacteria regarding their gene organization, which might lead to their differences in sporulation. For example, in B. subtilis, deletion of 6S-1 RNA accelerates the utilization of nutrients, leading to the early arrival of nutrient deprivation conditions, which in turn induces the earlier expression of key early sporulation-related genes, ultimately promoting the earlier sporulation (Wassarman, 2018), while deletion of 6S-1 RNA in B. thuringiensis resulted in suppression of sporulation. In order to explore its possible regulatory mechanism, we have determined the transcriptomes of BMB171 and $\triangle s s r S A B$ in the stationary phase. After analyses, we found that the transcription levels of sporulation-related genes did not change significantly between BMB171 and $\triangle s s r S A B$ (Supplementary Table S2), indicating that 6S RNA does not affect sporulation by directly regulating the temporal expression of sporulation-related genes. Moreover, the experiments of decoyinine-induced sporulation in the logarithmic phase (Figure 6) and RT-qPCR detection of the transcription levels of key early sporulation-related genes (Supplementary Figure S4) further proved this conclusion. Since there is no available transcriptomic data from the 6S-1 RNA deletion in B. subtilis, we are currently unable to compare the $6 \mathrm{~S}-1$ RNA regulatory mechanisms between the two bacteria in a more comprehensive way. Altogether, the $6 \mathrm{~S}$ RNAs of B. subtilis group and B. cereus group bacteria exhibited different gene organization and physiological functions, indicating that $6 \mathrm{~S}$ RNAs might regulate the biological functions of $B$. subtilis group and $B$. cereus group bacteria via different mechanisms.

Then, how does deletion of 6S-1 RNA inhibit sporulation of $B$. thuringiensis? After further analyzing the transcriptomic data, we found that the transcription levels of genes involved in carbohydrate transport and metabolism, nucleotide transport and metabolism, protein translation, and energy production and conversion decreased significantly in $\triangle s s r S A B$ (Supplementary Table S3). According to our previous report that sporulation and parasporal crystal formation do require a lot of material and energy supply in B. thuringiensis (Wang et al., 2013c), we therefore speculate that the insufficient supply of material and energy in $\triangle s s r S A B$ was the main reason for inhibition of sporulation.

How does deletion of 6S-1 RNA inhibits the parasporal crystal formation of $B$. thuringiensis? In crystalliferous strain BMB171-cry, cry1Ac10 with the original promoter was regulated by a sporulation-specific sigma factor SigE. In the transcriptomic data, we did not find a significant expression difference of sigE between BMB171 and $\triangle$ ssrSAB (Supplementary Table S2). Meanwhile, RT-qPCR assays confirmed that cry1Ac10 had no expression difference between BMB171 and $\triangle s s r S A B$ (Supplementary Figure S7). However, the content of parasporal crystals was significantly reduced in $\triangle s s r S A B$ (Figure 5B). This indicates that deletion of $6 \mathrm{~S}$ RNA may inhibit the translation of Cry1Ac10, further reducing the parasporal crystal formation.

Like 6S RNA, CsrA, CarD, and (p)ppGpp are all global regulatory factors that can respond to starvation stress in the stationary phase (Kalia et al., 2013; Romeo et al., 2013; Flentie et al., 2016). CsrA is believed to inhibit bacterial translation by interacting with conserved sequences on target mRNA under carbon starvation conditions (Romeo et al., 2013). Both CarD and (p)ppGpp, on the other hand, seem to regulate downstream gene transcription by interacting with RNA polymerase; in addition, the binding of CarD with RNA polymerase has been found to stabilize the transcription initiation complex to initiate the transcription of downstream genes (Flentie et al., 2016). Under starvation conditions, high levels of intracellular (p) ppGpp can also inhibit translation, which not only regulates the transcription of tRNA, rRNA, and ribosomal protein genes by binding to RNA polymerase, but also directly inhibits the bacterial translation activity through combining translation initiation factor IF2 with the translation elongation factors EF-Tu and EF-G (Kalia et al., 2013). Like CarD and (p)ppGpp, 6S RNA is also a global regulatory factor that can regulate gene transcription by binding to RNA polymerase. The consistent function of $6 \mathrm{~S}$ RNA, CsrA, CarD, and (p)ppGpp may be to maintain and optimize the survival rates of bacteria under different stress conditions, which may be manifested in rather complex metabolic regulation networks. Currently we cannot 
figure out what are the specific roles 6S RNA plays in these networks and also how they co-regulate the bacterial response to starvation stress in the stationary phase, which are issues deserve further exploration.

\section{DATA AVAILABILITY STATEMENT}

The original contributions presented in the study are included in the article/Supplementary Material, further inquiries can be directed to the corresponding authors.

\section{AUTHOR CONTRIBUTIONS}

$\mathrm{JH}$ and $\mathrm{ZL}$ designed the experiments. ZL, LZ, ZY, and LL did the experiments. JH, S-HC, JW, and ZL wrote and revised

\section{REFERENCES}

Ali, M. K., Li, X. F., Tang, Q., Liu, X. Y., Chen, F., Xiao, J. F., et al. (2017). Regulation of inducible potassium transporter KdpFABC by the KdpD/KdpE two-component system in Mycobacterium smegmatis. Front. Microbiol. 8:570. doi: 10.3389/fmicb.2017.00570

Brownlee, G. G. (1971). Sequence of 6S RNA of E. coli. Nat. New Biol. 229, 147-149. doi: 10.1038/newbio229147a0

Caulier, S. C., Nannan, C., Gillis, A., Licciard, F., Bragard, C., and Mahillon, J. (2019). Overview of the antimicrobial compounds produced by members of the Bacillus subtilis group. Front. Microbiol. 10:302. doi: 10.3389/fmicb.2019.00302

Cavanagh, A. T., and Wassarman, K. M. (2013). 6S-1 RNA function leads to a delay in sporulation in Bacillus subtilis. J. Bacteriol. 195, 2079-2086. doi: 10.1128/JB.00050-13

Cavanagh, A. T., and Wassarman, K. M. (2014). 6S RNA, a global regulator of transcription in Escherichia coli, Bacillus subtilis, and beyond. Annu. Rev. Microbiol. 68, 45-60. doi: 10.1146/annurev-micro-092611-150135

Craig, J. E., Ford, M. J., Blaydon, D. C., and Sonenshein, A. L. (1997). A null mutation in the Bacillus subtilis aconitase gene causes a block in Spo0Aphosphate-dependentgene expression. J. Bacteriol. 179, 7351-7359. doi: 10.1128/ jb.179.23.7351-7359.1997

Fayad, N., Awad, M. K., and Mahillon, J. (2019). Diversity of Bacillus cereus sensu lato mobilome. BMC Genomics 20:436. doi: 10.1186/s12864-019-5764-4

Flentie, K., Garner, A. L., and Stallings, C. L. (2016). Mycobacterium tuberculosis transcription machinery: ready to respond to host attacks. J. Bacteriol. 198, 1360-1373. doi: 10.1128/JB.00935-15

Fu, Y., Yu, Z. Q., Liu, S., Chen, B., Zhu, L., Li, Z., et al. (2018). C-di-GMP regulates various phenotypes and insecticidal activity of gram-positive Bacillus thuringiensis. Front. Microbiol. 9:45. doi: 10.3389/fmicb.2018.00045

He, J., Shao, X. H., Zheng, H. J., Li, M. S., Wang, J. P., Zhang, Q. Y., et al. (2010). Complete genome sequence of Bacillus thuringiensis mutant strain BMB171. J. Bacteriol. 192, 4074-4075. doi: 10.1128/JB.00562-10

Heilmann, B., Hakkila, K., Georg, J., Tyystjärvi, T., Hess, W. R., Axmann, I. M., et al. (2017). 6S RNA plays a role in recovery from nitrogen depletion in Synechocystis sp. PCC 6803. BMC Microbiol. 17:229. doi: 10.1186/ s12866-017-1137-9

Hindley, J. (1967). Fractionation of ${ }^{32} \mathrm{P}$-labelled ribonucleic acids on polyacrylamide gels and their characterization by fingerprinting. J. Mol. Biol. 30, 125-136. doi: 10.1016/0022-2836(67)90248-3

Hoch, P. G., Burenina, O. Y., Weber, M. H. W., Elkina, D. A., Nesterchuk, M. V., Sergiev, P. V., et al. (2015). Phenotypic characterization and complementation analysis of Bacillus subtilis 6S RNA single and double deletion mutants. Biochimie 117, 87-99. doi: 10.1016/j.biochi.2014.12.019

Ikehara, K., Okamoto, M., and Sugae, K. (1982). Induction of Bacillus subtilis sporulation by decoyinine and the concomitant disappearance of ppGpp the manuscript. All authors contributed to the article and approved the submitted version.

\section{FUNDING}

This paper is supported by the National Natural Science Foundation of China (grants 31770087 and 31970074), and the Cultivation fund project of Fujian Academy of Agricultural Sciences (AGP2018-2).

\section{SUPPLEMENTARY MATERIAL}

The Supplementary Material for this article can be found online at: https://www.frontiersin.org/articles/10.3389/fmicb.2020.604458/ full\#supplementary-material

in vegetative cells. J. Biochem. 91, 1089-1092. doi: 10.1093/oxfordjournals. jbchem.a133759

Janes, B. K., and Stibitz, S. (2006). Routine markerless gene replacement in Bacillus anthracis. Infect. Immun. 74, 1949-1953. doi: 10.1128/IAI.74.3.1949-1953.2006

Jiang, X., Diwa, A., and Belasco, J. G. (2000b). Regions of RNase E important for 5 '-end-dependent RNA cleavage and autoregulated synthesis. J. Bacteriol. 182, 2468-2475. doi: 10.1128/jb.182.9.2468-2475.2000

Jiang, M., Shao, W., Perego, M., and Hoch, J. A. (2000a). Multiple histidine kinases regulate entry into stationary phase and sporulation in Bacillus subtilis. Mol. Microbiol. 38, 535-542. doi: 10.1046/j.1365-2958.2000.02148.x

Kalia, D., Merey, G., Nakayama, S., Zheng, Y., Zhou, J., Luo, Y. L., et al. (2013). Nucleotide, c-di-GMP, c-di-AMP, cGMP, cAMP, (p)ppGpp signaling in bacteria and implications in pathogenesis. Chem. Soc. Rev. 42, 305-341. doi: $10.1039 / \mathrm{c} 2 \operatorname{cs} 35206 \mathrm{k}$

Kim, K. S., and Lee, Y. (2004). Regulation of 6S RNA biogenesis by switching utilization of both sigma factors and endoribonucleases. Nucleic Acids Res. 32, 6057-6068. doi: 10.1093/nar/gkh939

Li, L., Yang, C., Liu, Z., Li, F., and Yu, Z. (2000). Screening of acrystalliferous mutants from Bacillus thuringiensis and their transformation properties. Wei Sheng Wu Xue Bao 40, 85-90.

Liu, Y., Du, J., Lai, Q. L., Zeng, R. Y., Ye, D. Z., Xu, J., et al. (2017). Proposal of nine novel species of the Bacillus cereus group. Int. J. Syst. Evol. Microbiol. 67, 2499-2508. doi: 10.1099/ijsem.0.001821

Lopez, D., Vlamakis, H., and Kolter, R. (2009). Generation of multiple cell types in Bacillus subtilis. FEMS Microbiol. Rev. 33, 152-163. doi: 10.1111/j. 1574-6976.2008.00148.x

Mackie, G. A. (1998). Ribonuclease E is a 5'-end-dependent endonuclease. Nature 395, 720-723. doi: 10.1038/27246

Madhugiri, R., Pessi, G., Voss, B., Hahn, J., Sharma, C. M., Reinhardt, R., et al. (2012). Small RNAs of the Bradyrhizobium/Rhodopseudomonas lineage and their analysis. RNA Biol. 9, 47-58. doi: 10.4161/rna.9.1.18008

Mikulík, K., Bobek, J., Zídková, J., and Felsberg, J. (2014). 6S RNA modulates growth and antibiotic production in Streptomyces coelicolor. Appl. Microbiol. Biotechnol. 98, 7185-7197. doi: 10.1007/s00253-014-5806-4

Miller, R. A., Beno, S. M., Kent, D. J., Carroll, L. M., Martin, N. H., Boor, K. J., et al. (2016). Bacillus wiedmannii sp. nov., a psychrotolerant and cytotoxic Bacillus cereus group species isolated from dairy foods and dairy environments. Int. J. Syst. Evol. Microbiol. 66, 4744-4753. doi: 10.1099/ijsem.0.001421

Mitani, T., Heinze, J. E., and Freese, E. (1977). Induction of sporulation in Bacillus subtilis by decoyinine or hadacidin. Biochem. Biophys. Res. Commun. 77, 1118-1125. doi: 10.1016/s0006-291x(77)80094-6

Predich, M., Nair, G., and Smith, I. (1992). Bacillus subtilis early sporulation genes $k i n A, s p o 0 F$, and $s p o 0 A$ are transcribed by the RNA polymerase containing sigma H. J. Bacteriol. 174, 2771-2778. doi: 10.1128/JB.174.9.27 $71-2778.1992$ 
Qi, M. X., Mei, F., Wang, H., Sun, M., Wang, G. J., Yu, Z. N., et al. (2015). Function of global regulator CodY in Bacillus thuringiensis BMB171 by comparative proteomic analysis. J. Microbiol. Biotechnol. 25, 152-161. doi: 10.4014/jmb.1406.06036

Ren, J., Sang, Y., Qin, R., Cui, Z. L., and Yao, Y. F. (2017). 6S RNA is involved in acid resistance and invasion of epithelial cells in Salmonella enterica serovar Typhimurium. Future Microbiol. 12, 1045-1057. doi: 10.2217/ fmb-2017-0055

Romeo, T., Vakulskas, C. A., and Babitzke, P. (2013). Posttranscriptional regulation on a global scale: form and function of Csr/Rsm systems. Environ. Microbiol. 15, 313-324. doi: 10.1111/j.1462-2920.2012.02794.x

Steuten, B., Schneider, S., and Wagner, R. (2014). 6S RNA: recent answersfuture questions. Mol. Microbiol. 91, 641-648. doi: 10.1111/mmi.12484

Tang, Q., Yin, K., Qian, H. L., Zhao, Y. W., Wang, W., Chou, S. H., et al. (2016). Cyclic di-GMP contributes to adaption and virulence of Bacillus thuringiensis through a riboswitch-regulated collagen adhesion protein. Sci. Rep. 6:28807. doi: 10.1038/srep28807

Tock, M. R., Walsh, A. P., Carroll, G., and McDowall, K. J. (2000). The CafA protein required for the 5 -maturation of $16 \mathrm{~S}$ rRNA is a 5 -end-dependent ribonuclease that has context-dependent broad sequence specificity. J. Biol. Chem. 275, 8726-8732. doi: 10.1074/jbc.275.12.8726

Trotochaud, A. E., and Wassarman, K. M. (2004). 6S RNA function enhances long-term cell survival. J. Bacteriol. 186, 4978-4985. doi: 10.1128/JB.186.15. 4978-4985.2004

Wang, J. P., Ai, X. L., Mei, H., Fu, Y., Chen, B., Yu, Z. N., et al. (2013a). High-throughput identification of promoters and screening of highly active promoter-5'-UTR DNA region with different characteristics from Bacillus thuringiensis. PLoS One 8:e62960. doi: 10.1371/journal.pone.0062960

Wang, X., Cai, X., Ma, H. D., Yin, W., Zhu, L., Li, X. F., et al. (2019). A c-di-AMP riboswitch controlling $k d p F A B C$ operon transcription regulates the potassium transporter system in Bacillus thuringiensis. Commun. Biol. 2:151. doi: 10.1038/s42003-019-0414-6

Wang, X., Li, Z., Li, X., Qian, H. L., Cai, X., Li, X. F., et al. (2016). Poly- $\beta$ hydroxybutyrate metabolism is unrelated to the sporulation and parasporal crystal protein formation in Bacillus thuringiensis. Front. Microbiol. 7:836. doi: $10.3389 /$ fmicb. 2016.00836
Wang, J. P., Mei, H., Qian, H. L., Tang, Q., Liu, X. C., Yu, Z. N., et al. (2013b). Expression profile and regulation of spore and parasporal crystal formationassociated genes in Bacillus thuringiensis. J. Proteome Res. 12, 5487-5501. doi: $10.1021 / \mathrm{pr} 4003728$

Wang, J., Mei, H., Zheng, C., Qian, H. L., Cui, C., Fu, Y., et al. (2013c). The metabolic regulation of sporulation and par-asporal crystal formation in Bacillus thuringiensis revealed by transcriptomics and proteomics. Mol. Cell. Proteomics 12, 1363-1376. doi: 10.1074/mcp.M112.023986

Wassarman, K. M. (2018). 6S RNA, a global regulator of transcription. Microbiol. Spectr. 6:RWR-0019-2018. doi: 10.1128/microbiolspec.RWR-0019-2018

Wassarman, K. M., and Storz, G. (2000). 6S RNA regulates E. coli RNA polymerase activity. Cell 101, 613-623. doi: 10.1016/s0092-8674(00)80873-9

Wehner, S., Damm, K., Hartmann, R. K., and Marz, M. (2014). Dissemination of 6S RNA among bacteria. RNA Biol. 11, 1467-1478. doi: 10.4161/rna.29894

Zheng, C., Ma, Y., Wang, X., Xie, Y. Q., Ali, M. K., and He, J. (2015). Functional analysis of the sporulation-specific diadenylate cyclase CdaS in Bacillus thuringiensis. Front. Microbiol. 6:908. doi: 10.3389/fmicb.2015.00908

Zheng, C., Yu, Z. Q., Du, C. Y., Gong, Y. J., Yin, W., Li, X. F., et al. (2020). 2-Methylcitrate cycle: a well-regulated controller of Bacillus sporulation. Environ. Microbiol. 22, 1125-1140. doi: 10.1111/1462-2920.14901

Zhou, H., Zheng, C., Su, J. M., Chen, B., Fu, Y., Xie, Y. Q., et al. (2017). Characterization of a natural triple-tandem c-di-GMP riboswitch and application of the riboswitch-based dual-fluorescence reporter. Sci. Rep. 6:20871. doi: 10.1038/srep20871

Conflict of Interest: The authors declare that the research was conducted in the absence of any commercial or financial relationships that could be construed as a potential conflict of interest.

Copyright (c) $2020 \mathrm{Li}, \mathrm{Zhu}, \mathrm{Yu}$, Liu, Chou, Wang and He. This is an open-access article distributed under the terms of the Creative Commons Attribution License (CC BY). The use, distribution or reproduction in other forums is permitted, provided the original author(s) and the copyright owner(s) are credited and that the original publication in this journal is cited, in accordance with accepted academic practice. No use, distribution or reproduction is permitted which does not comply with these terms. 